\title{
Small bowel feeding versus gastric feeding in critically ill adults: more attention should be paid to specific populations
}

\author{
Wan-Jie Gu and Jing-Chen Liu* \\ See related research by Deane et al. http://ccforum.com/content/17/3/R125, Alhazzani et al., http://ccforum.com/content/17/4/R127 and \\ Chang et al., http://ccforum.com/content/17/3/R118
}

As all of us are well aware, there are a lot of unanswered questions in the nutrition support of patients, especially in the care of critically ill adults. Despite extensive studies, however, the optimal route (small bowel feeding versus gastric feeding) of enteral nutrition remains debated.

In recent issues of Critical Care, three meta-analyses focus on the topic [1-3]. Deane and colleagues [1] and Alhazzani and colleagues [2] both concluded that small intestinal feeding was superior to gastric feeding in reducing the risk of pneumonia in critically ill patients. We performed a subgroup analysis based on the analysis by Deane and colleagues [1], and the results suggested that small bowel feeding was associated with a reduction in the incidence of pneumonia in trauma patients (relative risk (RR) $0.67,95 \%$ confidence interval (CI) 0.52 to 0.87 ;
$P=0.003)$ but no reduction in the medical or surgical ICU population or both (RR $0.86,95 \%$ CI 0.58 to 1.26 ; $P=0.43$ ). Moreover, in another meta-analysis focusing on patients with severe acute pancreatitis, the authors suggested that small intestinal feeding was not superior to gastric feeding [3].

As implied above, what is noteworthy is that the effects of small bowel versus gastric feeding on clinical outcomes may vary in different population groups. In this case, we were unable to demonstrate a clinical benefit from small bowel versus gastric feeding in a mixed group of critically ill patients. More attention should be paid to specific populations, such as medical or surgical ICU patients (or both), patients with severe acute pancreatitis, and trauma patients.

\section{Authors' response \\ Yu-sui Chang, Hua-qun Fu and Ji-chun Liu}

Whether the gastric or the postpyloric feeding route is more beneficial to critically ill patients receiving enteral nutrition remains controversial. Recently, some studies have suggested that nasojejunal nutrition was not superior to nasogastric nutrition with respect to energy delivery and frequency of pneumonia in critically ill patients. However, in two meta-analyses focusing on the route of enteral feeding in critically ill patients, the authors demonstrated that small bowel feeding was associated with reduced risk of pneumonia [1,2] and improved nutrient intake [1]. However, in the above Letter, $\mathrm{Gu}$ and Liu showed that small bowel feeding was associated with a reduced frequency of pneumonia in trauma patients but not in a medical or surgical ICU population (or both)

\footnotetext{
* Correspondence: jingchenliu1964@sina.cn

Department of Anaesthesiology, The First Affiliated Hospital, Guangxi Medical University, 22 Shuangyong Road, Nanning, Guangxi 530021, China
}

after a subgroup analysis based on the analysis by Deane and colleagues [1] was performed. Moreover, Chang and colleagues [3] suggested that nasogastric feeding was not inferior to nasojejunal feeding in patients with predicted severe acute pancreatitis. These findings may imply that the efficacy and nutritional risk of small intestine or gastric feeding (or both) may differ among different subgroups of critically ill patients. Severity and pathophysiology of illness are closely related to secretion of stress hormones and influence gastrointestinal motility.

Of course, the limitations, including a small total sample size, in these studies should be taken into account because small studies may overestimate the effect sizes in critical care meta-analyses and lead to erroneous conclusions [4]. Thus more attempts to evaluate whether there is a clinical difference in gastric feeding versus small intestine feeding in specific populations are warranted. 


\section{Abbreviations}

$\mathrm{Cl}$ : Confidence interval; RR: Relative risk.

\section{Competing interests}

The authors declare that they have no competing interests.

\section{Authors' contributions}

W-JG and J-CL conceived the study, participated in the design, collected the data, and drafted the manuscript. Both authors read and approved the final manuscript.

Published: 23 Oct 2013

\section{References}

1. Deane AM, Dhaliwal R, Day AG, Ridley EJ, Davies AR, Heyland DK: Comparisons between intragastric and small intestinal delivery of enteral nutrition in the critically ill: a systematic review and metaanalysis. Crit Care 2013, 17:R125.

2. Alhazzani W, Almasoud A, Jaeschke R, Lo BW, Sindi A, Altayyar S, FoxRobichaud A: Small bowel feeding and risk of pneumonia in adult critically ill patients: a systematic review and meta-analysis of randomized trials. Crit Care 2013, 17:R127.

3. Chang Y, Fu H, Xiao Y, Liu J: Nasogastric or nasojejunal feeding in predicted severe acute pancreatitis: a meta-analysis. Crit Care 2013, 17:R118.

4. Zhang Z, Xu X, Ni H: Small studies may overestimate the effect sizes in critical care meta-analyses: a meta-epidemiological study. Crit Care 2013, 17:R2.

$10.1186 /$ cc13079

Cite this article as: Gu and Liu: Small bowel feeding versus gastric feeding in critically ill adults: more attention should be paid to specific populations. Critical Care 2013, 17:462 